\title{
Sars-Cov-2 e Injúria Miocárdica com Supradesnivelamento de ST sem Doença Coronariana: Relato de Caso e Breve Revisão da Literatura
}

SARS-CoV-2 and Myocardical Injury with ST-Elevation without Coronary Disease

Carolina Ragonetti, ${ }^{1}$ Enzo Oku Martinazzo, ${ }^{1 \oplus}$ Felipe Montesano Fazionato, ${ }^{1}$ Guilherme Osório Guimarães Ferreira, ${ }^{1}$ Milena Piccolo Santana, ${ }^{1}$ Camila Hartmann ${ }^{1,2}$

Pontifícia Universidade Católica do Paraná, ${ }^{1}$ Curitiba, $P R$ - Brasil

Hospital Marcelino Champagnat, ${ }^{2}$ Curitiba, $P R$ - Brasil

\section{Introdução}

Durante a pandemia de SARS-CoV-2, há relatos de infecção cursando com injúria miocárdica aguda (IMA), que causa piores resultados clínicos. A manifestação pode ser com elevação de troponina, alterações de imagem e eletrocardiográficas. ${ }^{1}$ Nesse sentido, a IMA com supradesnivelamento de ST (SST) tem sido observada em alguns pacientes. Contudo, apesar de alteração isquêmica ao eletrocardiograma (ECG), os exames podem não evidenciar obstrução, não sendo oclusão coronariana a causa da injúria. ${ }^{2}$

\section{Relato de Caso}

Masculino, 42 anos de idade, sem comorbidades prévias, admitido em hospital de Curitiba-PR com queixa de tosse seca há 6 dias e odinofagia há 2 dias, com piora no dia anterior, apresentando tosse com secreção amarelada, dispneia, mal-estar, febrícula, mialgia e cefaleia. Contato recente com pacientes positivos para SARS-CoV-2. Exame físico: bom estado geral, corado, hidratado, eupneico, FC 100bpm, FR 18mrpm, $\mathrm{SpO}_{2} 98 \%$, temperatura $36,2^{\circ} \mathrm{C}$ e PA $226 / 158 \mathrm{mmHg}$. Ausculta pulmonar com crepitantes no terço inferior de hemitórax esquerdo (HTE) e cardíaca sem alterações. Controle da pressão arterial (PA) feito com nitroglicerina e solicitação de exames.

Diante de troponina I $76,1 \mathrm{pg} / \mathrm{mL}(\mathrm{VR}<2,3 \mathrm{pg} / \mathrm{mL})$, foi realizado ECG (Figura 1), evidenciando ritmo sinusal, SST em V1 a V3 e sobrecarga de ventrículo esquerdo (VE). Paciente relatou que, na noite anterior, houve episódios de dor em pontada em HTE de poucos minutos.

A cineangiocoronariografia (Figura 2) evidenciou disfunção segmentar de VE e ausência de trombos ou processo aterosclerótico significativo em coronárias.

\section{Palavras-chave}

Pandemia; SARS-CoV-2; Coronavirus-19; Miocardite/ complicações; Eletrocardiografia/métodos; Cardiomiopatia de Takotsubo; Angiografia Coronária/métodos.

Correspondência: Enzo Oku Martinazzo •

Pontifícia Universidade Católica do Paraná - Rua Imac. Conceição, 1155.

CEP 80215-901, Prado Velho, Curitiba, PR - Brasil

E-mail: enzo.martinazzoo@gmail.com

Artigo recebido em 28/11/2020, revisado em 28/01/2021, aceito em 24/02/2021

DOI: https://doi.org/10.36660/abc.20201268
No D2, já na UTI, foi iniciado hidralazina, nitrato, anlodipino e carvedilol para desmame da nitroglicerina e controle da PA. Foi necessário uso de cateter de $\mathrm{O}_{2}$ nasal 3L/min e início de ceftriaxona, azitromicina e dexametasona.

Tomografia: opacidades pulmonares consolidativas, broncograma aéreo e vidro fosco periférico associados a densificações subpleurais no lobo inferior esquerdo. Ecocardiograma: VE aumentado com padrão de hipertrofia concêntrica importante e função sistólica moderadamente comprometida. Átrio esquerdo (AE) aumentado, discreto refluxo mitral, tricúspide e aórtico e ectasia da raiz da aorta.

RT-PCR positivo para SARS-CoV-2. Levantadas hipóteses diagnósticas de miocardite associada ao SARS-CoV-2, trombose com lise espontânea, lesão microvascular, insuficiência cardíaca (IC) por miocardiopatia hipertensiva ou de Takotsubo. Recebeu alta com tratamento otimizado para IC. Em retorno após 60 dias, RNM cardíaca (Figura 3) evidenciou: dilatação da cavidade de VE associada a disfunção sistólica global importante (FEVE 23\%), dilatação da cavidade do ventrículo direito associada a disfunção sistólica global leve (FEVD 43\%), hipertrofia excêntrica do VE, AE dilatado e ausência de necrose.

\section{Discussão}

Neste relato, a IMA, evidenciada por SST e troponina elevada, pode ter diversas explicações: uma das hipóteses é a ocorrência de lesão miocárdica direta pelo vírus (miocardite). Contudo, a RM não demonstrou padrão de fibrose mesocárdica, edema ou necrose, não corroborando essa hipótese. Pelo fato de o diagnóstico ter sido tardio e a cineangiocoronariografia não ter evidenciado trombos ou processo aterosclerótico, outra possibilidade é ocorrência de trombose com lise espontânea ou lesão microvascular - a hipercoagulabilidade vista no estado pró-inflamatório na Covid-19 predispõe eventos coronarianos agudos., ${ }^{1,3}$ Outra hipótese é cardiomiopatia Takotsubo-like, que pode ocorrer em pacientes com SARS-CoV-2. ${ }^{4}$ Entretanto, a ventriculografia realizada com a cineangiocoronariografia e o ecocardiograma não evidenciaram padrão dessa cardiomiopatia, que poderia ser excluída caso a miocardite fosse confirmada. ${ }^{5}$ Por fim, há possibilidade de associação de alguma hipótese anterior com cardiomiopatia hipertensiva: apresentou pico hipertensivo e provável hipertensão não diagnosticada.

Quanto à fisiopatologia da Covid-19, há a ligação da proteína spike do vírus ao receptor da ECA-2, após a TMPRSS2 ativar a spike. ${ }^{3}$ Então, ocorre entrada do SARS-CoV-2 nas células 


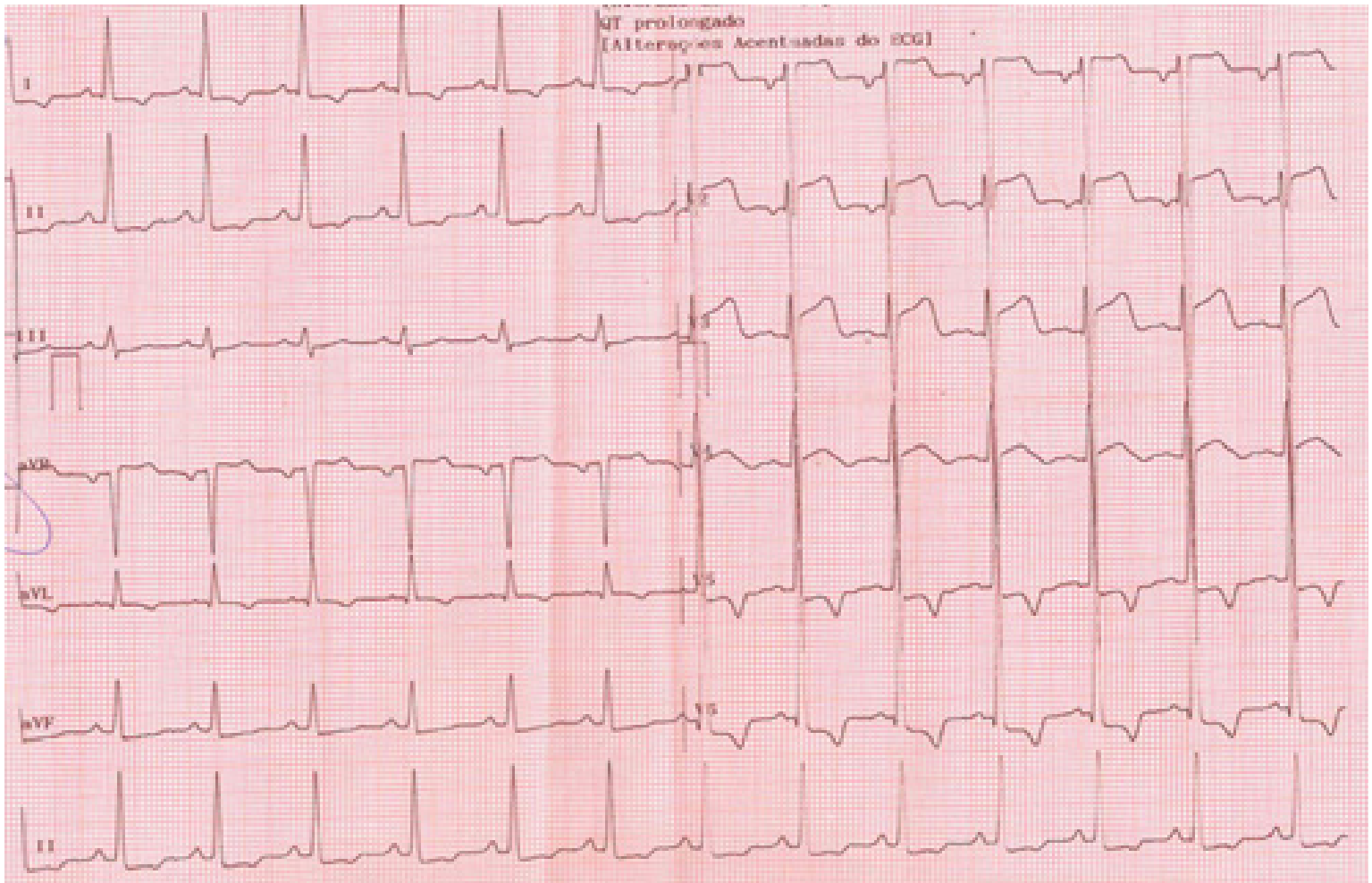

Figura 1 - Eletrocardiograma de admissão.

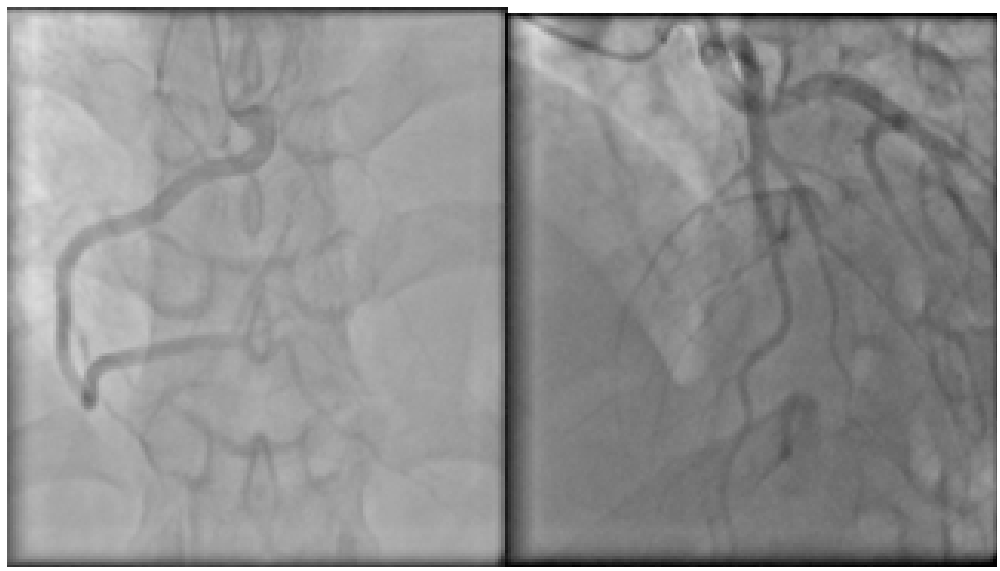

Figura 2 - Cineangiocoronariografia de artéria coronária direita e artéria descendente anterior.

através da ECA-2, presente em múltiplos tecidos do organismo, incluindo cardiomiócitos. Essa enzima converte angiotensina II, um componente inflamatório, vasoconstritor, oxidativo e fibrótico, em angiotensina (1-7) , de ações opostas. Portanto, ocorrem duas principais situações: entrada do vírus em células miocárdicas e, uma vez que os receptores estão bloqueados por proteínas virais, acúmulo de angiotensina II, além de liberação massiva de citocinas. ${ }^{6-8}$
Além disso, estudos mostram que a IMA pode ocorrer na Covid-19 por isquemia miocárdica ou processo não isquêmico. A lesão é relacionada a quadros mais graves da doença, como desenvolvimento de IC em até $23 \%$ dos pacientes. ${ }^{9} \mathrm{Na}$ China, estudos mostram que até $17 \%$ dos pacientes apresentaram elevação da troponina. ${ }^{7,8}$

A elevação de troponina em quadro não isquêmico de injúria miocárdica pode ser explicada por hipóxia tecidual, 


\section{Carta Científica}

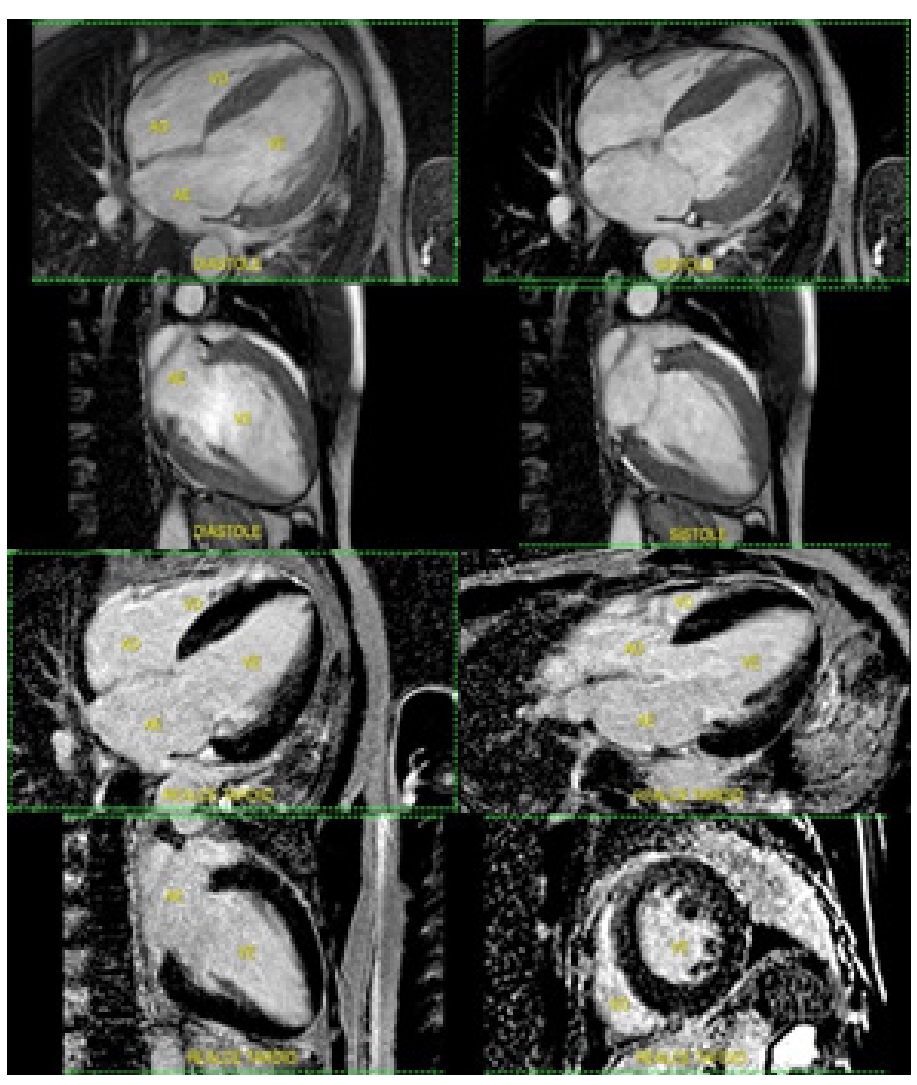

Figura 3 - Ressonância magnética cardiaca com estudo dinâmico (superior) e realce tardio (inferior).

sepse, resposta inflamatória sistêmica, tromboembolismo venoso e estresse miocárdico. ${ }^{8}$ Havendo obstrução, a hipótese é que o vírus possa causar instabilidade e hemorragias intraplaca, gerando exposição do colágeno, lesão microvascular e formação de trombos. ${ }^{1,3,8} \mathrm{Na}$ ausência de processo aterosclerótico, acredita-se que o desbalanço entre oferta e consumo de oxigênio cause um infarto agudo do miocárdio (IAM) tipo 2. ${ }^{3}$ Além dos mecanismos de lesão miocárdica diretos, há mecanismos indiretos: tempestade de citocinas e Takotsubo. Essa cardiomiopatia representa até 3\% das suspeitas de síndrome coronariana aguda, e sabe-se que situações de infecção respiratória, estresse emocional e físico podem ser gatilhos, cursando com disfunção transitória do VE. ${ }^{3,5,7}$

Comparando com casos semelhantes (Tabela 1), Aragão et al., ${ }^{2}$ descreveram elevação de troponina, mas difere do nosso paciente pela ausência de IC constatada pela redução significativa da fração de ejeção do ventrículo esquerdo (FEVE). Inciardi et al., ${ }^{10}$ também descreveram redução da FEVE, porém, mais branda. Já, Huyut ${ }^{11}$ não mostra elevação da troponina, mas a redução transitória da FEVE sugere ocorrência de miocardiopatia. ${ }^{11}$

Stefanini et al., ${ }^{12}$ demostraram que $85,7 \%$ dos pacientes de uma série de casos tinham sinais de infarto com SST como primeira manifestação sintomática da Covid-19, e que $39,3 \%$ não apresentavam doença obstrutiva. Nosso paciente teve SST, mas não como primeira manifestação, além de não ter apresentado oclusão na cineangiocoronariografia.
Assim como a maioria dos pacientes do estudo, o nosso seguiu padrão de prognóstico benigno. ${ }^{12}$

\section{Conclusão}

Neste relato, apresentamos um caso atípico de manifestação cardiológica da Covid-19, em que houve SST sem evidência de coronariopatia, evoluindo para IC com fração de ejeção reduzida. Como discutido, as hipóteses de miocardite viral, trombose com lise espontânea, lesão microvascular, Takotsubo e cardiomiopatia hipertensiva não foram completamente estabelecidas, podendo até coexistir. Por fim, enaltecemos que o esclarecimento dos mecanismos envolvidos visa à identificação precoce e ao manejo adequado dos pacientes, acarretando melhora dos desfechos e compreensão das possíveis sequelas.

\section{Contribuição dos autores}

Concepção e desenho da pesquisa: Martinazzo EO; Obtenção de dados; Análise e interpretação dos dados; Redação do manuscrito; e Revisão crítica do manuscrito quanto ao conteúdo intelectual importante: Ragonetti C, Martinazzo EO, Fazionato FM, Ferreira GOG, Santana MP, Hartmann C.

\section{Potencial conflito de interesse}

Não há conflito com o presente artigo 


\begin{tabular}{|c|c|c|c|c|}
\hline Casos & Curitiba & Aragão et al. ${ }^{2}$ & Inciardi et al. ${ }^{10}$ & Huyut $^{11}$ \\
\hline Idade/sexo & 42/masculino & 39/masculino & 53/feminino & 59/feminino \\
\hline FEVE & $23 \%$ & $62 \%$ & $40 \%$ & $52 \%$ \\
\hline Troponina I & $76,1 \mathrm{pg} / \mathrm{mL}$ & $25,20 \mathrm{ng} / \mathrm{mL}$ & $0,89^{*}$ & Normal \\
\hline ECG & SST & SST & SST & Sem alteração \\
\hline RT-PCR SARS-CoV-2 & Positivo & Positivo & Positivo & Positivo \\
\hline Hipocinesia & Difusa & $\begin{array}{l}\text { Segmento médio parede } \\
\text { anterosseptal }\end{array}$ & Difusa & - \\
\hline
\end{tabular}

* Troponina T ultrassensível. FEVE: fração de ejeção do ventrículo esquerdo; ECG: eletrocardiograma.

\section{Fontes de financiamento}

O presente estudo não teve fontes de financiamento externas.

\section{Vinculação acadêmica}

Não há vinculação deste estudo a programas de pósgraduação.

\section{Referências}

1. Schiavone M, Gobbi C, Biondi-Zoccai G,D'SAscenzo F, Palazzioli A, Gasparetti A, et al. Acute coronary syndromes and Covid-19: exploring the uncertainties. J Clin Med. 2020 Jun; 9(6):1683.

2. Aragão RC de A, Alves MC, Passos HD, Gonçalves FG, Baumworcel L, Barreto Filho JA. Lesão miocárdica na Covid-19: um desafio para o cardiologista clínico. Arq Bras Cardiol. 2020; 115(1):139-41.

3. Albuquerque J, Neto DF, Marcondes-Braga FG, Figueiredo Neto JA,Marcondes-Braga,F, Moura LZ,Figueiredo ALS, et al. Coronavirus e o miocardio:revisão. Arq Bras Cardiol.2020;114(6):1051-7;

4. Singh S, Desai R, Gandhi Z,Fong HK, Dores Wamy S, Desai V. et al. Takotsubo syndrome in patients with Covid-19: a systematic review of published cases. SN Compr Clin Med. 2020; 2(11):2102-8.

5. Ghadri J, Wittstein IS, Prasad A , Sharkey S, Dote K, Akashi YJ, et al. International expert consensus document on Takotsubo syndrome (part I): clinical characteristics, diagnostic criteria, and pathophysiology. Eur Heart J. 2018; 39(22):2032-46.

6. Çınar T, Hayıroğlu Mi, Çiçek V,Uzun M, Orhan AL. Covid-19 and acute myocarditis: current literature review and diagnostic challenges. Rev Assoc Med Bras. 2020; 66 2(Suppl 2):48-54.

7. Siripanthong B, Nazarian S, Muser D, Deor R, Santangeli P, Khanji M, et al. Recognizing Covid-19-related myocarditis: the possible pathophysiology

\section{Aprovação ética e consentimento informado}

Este estudo foi aprovado pelo Comitê de Ética da Pontifícia Universidade Católica do Paraná sob o número de protocolo 30188020.7.1001.0020. Todos os procedimentos envolvidos nesse estudo estão de acordo com a Declaração de Helsinki de 1975, atualizada em 2013. and proposed guideline for diagnosis and management. Heart Rhythm. 2020; 17(9):1463-71.

8. Imazio M, Klingel K, Kindermann I, Brucato A, Rosa FG, Adler Y, et al Covid-19 pandemic and troponin: indirect myocardial injury, myocardial inflammation or myocarditis? Heart. 2020 Aug 1; 106(15):1127-31. [Internet] Disponível em: http://heart.bmj.com/content/106/15/1127. abstract.

9. Driggin E, Madhavan MV, Bikdeli B,CHuich T, Laracy J, Biondi-Zoccai $\mathrm{G}$, et al. et al. Cardiovascular considerations for patients, health care workers, and health systems during the Covid-19 pandemic. J Am Coll Cardiol. 2020; 75(18):2352-71.

10. Inciardi RM, Lupi L, Zaccone G, Italia L, Raffo M, Tomasoni D, et al. Cardiac involvement in a patient with coronavirus disease 2019 (Covid-19). JAMA Cardiol. 2020; 5(7):819-24.

11. Huyut MA. Nova pneumonia por coronavírus e miocardiopatia: relato de caso. Arq Bras Cardiol. 2020; 114(5):843-5.

12. Stefanini GG, Montorfano M, Trabattoni D,Andreini D, Ferrante G, Ancona M, et al. ST-Elevation myocardial infarction in patients with Covid-19: clinical and angiographic outcomes. Circulation. 2020;141(25); 2113-6. 